\title{
Nuevos datos sobre la flora amenazada de la Zona Especial de Conservación Río Zadorra / Zadorra Ibaia (Vitoria-Gasteiz).
}

\section{New data on threatened flora in the Special Area of Conservation River Zadorra / Zadorra Ibaia (Vitoria-Gasteiz).}

Asier Jáñez ${ }^{1}$, Agustí Agut ${ }^{1}$

\section{Resumen}

El tramo de la Zona Especial de Conservación Río Zadorra / Zadorra lbaia (ES2110010) que transcurre por el municipio de Vitoria-Gasteiz alberga 7 especies de flora vascular incluidas en el Catálogo Vasco de Especies Amenazadas.

Se ha actualizado, mediante cartografía y censos poblacionales, el conocimiento de estas especies y su estado de conservación, datos imprescindibles a partir de los cuales se fijarán las estrategias de conservación tanto ex situ como in situ del Banco de Germoplasma Vegetal del Jardín Botánico de Olarizu.

El trabajo ha permitido realizar censos directos de las poblaciones de Ranunculus auricomus, Pentaglottis sempervirens e Himantoglossum hircinum, a la vez que ha permitido censar y conocer cómo Berula erecta y Narcissus pallidiflorus poseen una mayor área de distribución que la conocida inicialmente. Por último, se citan por primera vez nuevas localidades de Narcissus minor subsp. exiguus y Narcissus x petri-mariae en el área de estudio, novedades para el conocimiento de la flora del municipio de Vitoria-Gasteiz.

Palabras clave: Catálogo Vasco Especies Amenazadas, censo, poblaciones, conservación.

1 Banco de Germoplasma Vegetal del Jardín Botánico de Olarizu

Dpto. de Medio Ambiente y Espacio Público del

Ayuntamiento de Vitoria-Gasteiz

Casa de la Dehesa de Olarizu s/n. 01006. Vitoria-Gasteiz

(Álava/Araba). País Vasco. España

*Correspondencia: ajplanez@gmail.com 


\begin{abstract}
The section of the Special Area of Conservation River Zadorra / Zadorra Ibaia (ES2110010) that runs through the municipality of Vitoria-Gasteiz, contains 7 species of vascular flora included in the Basque Catalogue of Threatened Species.

Through the use of cartography and population censuses, information on these species and their conservation status has been brought up to date. This data is essential in order to determine the ex situ and in situ conservation strategies of the Plant Germplasm Bank of the Olarizu Botanic Gardens.

The work has allowed direct censuses to be made of the populations of Ranunculus auricomus, Pentaglottis sempervirens and Himantoglossum hircinum, and has revealed that the distribution areas and populations of Berula erecta and Narcissus pallidiflorus are larger than previously estimated. Lastly, new sites for Narcissus minor subsp. exiguus and Narcissus $x$ petri-mariae have been recorded for the first time in a survey of the flora of the municipality of Vitoria-Gasteiz.
\end{abstract}

Key words: Basque Catalogue of Threatened Species, census, populations, conservation.

\title{
Laburpena
}

Gasteizko udalerritik igarotzen den Zadorra Ibaiko Kontserbazio Eremu Bereziko (ES2110010) tartean Euskal Autonomi Erkidegoko Flora mehatxatuaren Katalogoan agertzen diren 7 espezie aurki daitezke.

Kartografia eta errolda bidez, eguneratu egin dira espezie horien ezagutza eta beraien kontserbazio egoera, Olarizuko Lorategi Botanikoko Germoplasma Bankuaren ex situ eta in situ kontserbazio estrategiak finkatzeko datu garrantzitsuak hauek.

Alde batetik, lanari esker, Ranunculus auricomus, Pentaglottis sempervirens eta Himantoglossum hircinum-en populazioak erroldatu dira. Bestetik, erroldatzeaz gain, jakin da Berula erecta-k eta Narcissus pallidiflorus-ek duten banaketa lehendik ezagutzen zena baino handiagoa dela.

Azkenik, ikerketa eremuan bi espezieren kokagune berriak aurkitu dira, Narcissus minor subsp. exiguus eta Narcissus x petri-mariae, Vitoria-Gasteiz udalerriko flora hobeki ezagutzeko ekarpena.

Gako hitzak: Euskal Autonomi Erkidegoko Espezie Mehatxatuen Katalogoa, errolda, populazioak, kontserbazioa.

$$
\text { r. } 2 x
$$


El río Zadorra atraviesa el Territorio Histórico de Álava de norte a sur, y representa una de las principales arterias fluviales de este territorio. Se localiza en la parte occidental de esta provincia, en el Área Funcional de Álava Central, entre las regiones biogeográficas eurosiberiana y mediterránea. En virtud del Acuerdo 122/2015, del Consejo de Diputados de 10 de Marzo, que aprobó definitivamente el documento Directrices y medidas de gestión para la conservación favorable y para evitar el deterioro de hábitats y alteraciones sobre las cinco Zonas Especiales de Conservación en ríos del Territorio Histórico de Álava, fue declarada la Zona Especial de Conservación ES2110010 Zadorra Ibaia/ Río Zadorra (en adelante ZEC).

Este espacio, en el que se incluyen 13 hábitats de interés comunitario, queda integrado por el río Zadorra, desde su salida del embalse de Ullibarri-Gamboa hasta su desembocadura en el río Ebro (exceptuando el tramo fluvial que transcurre por el Condado de Treviño, ZEC Riberas del Zadorra ES4120051), y el río Santa Engracia, desde su salida del embalse de Urrunaga hasta la confluencia entre ambos. Del total de su superficie (334.07 ha), el 33,01\% (110.3 ha) corresponde al municipio de Vitoria-Gasteiz (Gobierno Vasco, 2014, Figura 1).

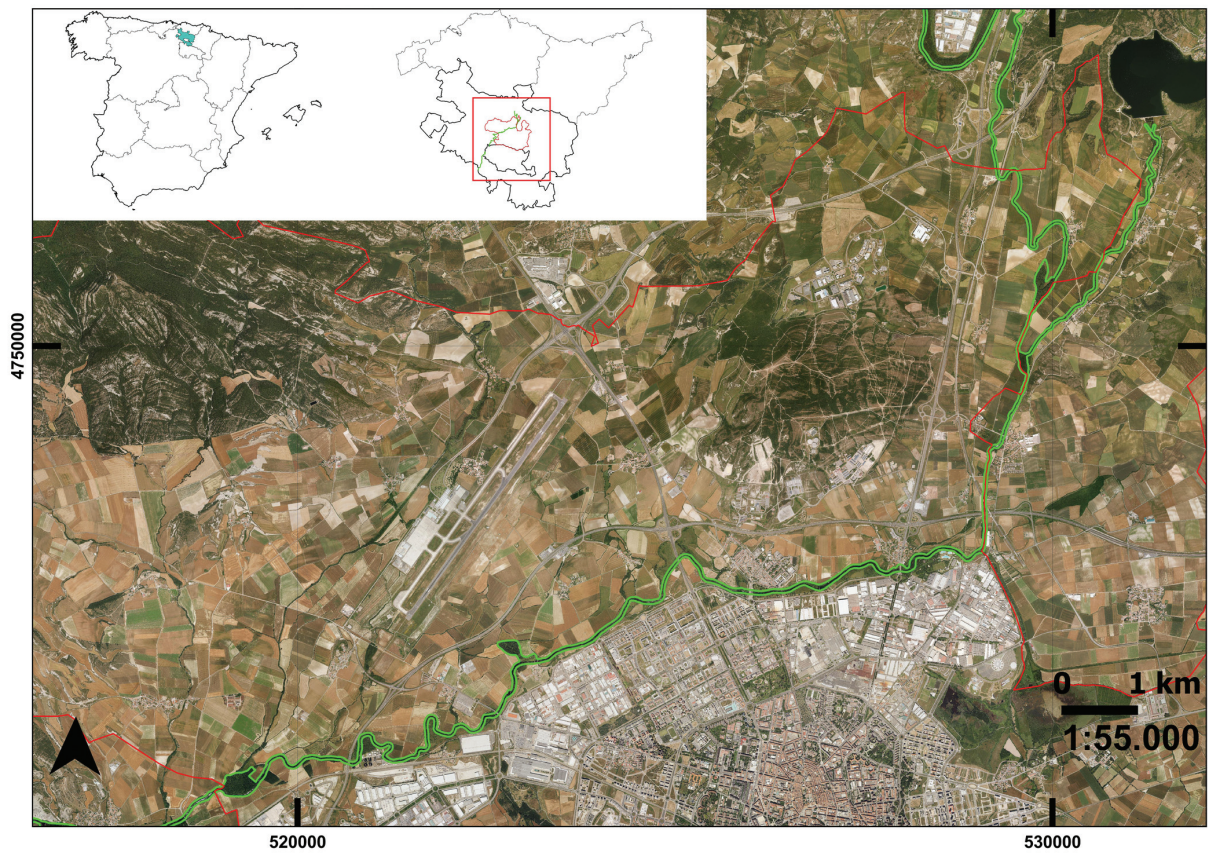

Fig. 1.- Localización del área de estudio. En verde: Zona Especial de Conservación Río Zadorra. En rojo: límite del municipio de Vitoria-Gasteiz.

Fig. 1.- Site of the study area. In green: Special Area of Conservation River Zadorra; in red: boundary of the municipality of Vitoria-Gasteiz. 
En el Documento de información ecológica y objetivos de conservación de la ZEC (Gobierno Vasco, 2014) se considera que ésta alberga en toda su extensión las siguientes 7 especies de flora vascular incluidas en el Catálogo Vasco de Especies Amenazadas (de ahora en adelante CVEA, BOPV/EHAA, 2011) (sin tener en cuenta Ruscus aculeatus, que no presenta problemas de conservación): Berula erecta (Hudson) Coville, Himantoglossum hircinum (L.) Sprengel, Narcissus minor L. subsp. exiguus (Salisb) Agut, Garaikoetxea, González, Jáñez, Solis \& Urrutia (syn. N.asturiensis subsp. jacetanus), Narcissus pseudonarcissus L. subsp. pallidiflorus (Pugsley) A. Fernández, Pentaglottis sempervirens (L.) L.H. Bailey, Ranunuculus auricomus L. y la extinta Nymphaea alba L., que no ha vuelto a ser observada en la ZEC desde hace más de 40 años (Figura 2, Tabla 1).

En el ámbito de la ZEC, merece una mención especial el robledal de Amarita, al borde del río Santa Engracia y a caballo entre las localidades de Amarita y Retana (Municipio de Vitoria-Gasteiz), enclave relicto del robledal de fondo de valle (Crataego laevigatae-Quercetum roboris) que otrora debió ocupar la mayor parte de la Llanada Alavesa (Gobierno Vasco, 2014; Uribe-Echebarría, 2010b) y en el cual se concentran las poblaciones de Pentaglottis sempervirens, Ranunculus auricomus y Narcissus pallidiflorus. A su vez, en este robledal se localiza Narcissus x petri-mariae, especie que pese a no estar incluida en el CVEA, merece ser incluida en el presente estudio debido a su rareza y el bajo número de localidades conocidas en la Comunidad Autónoma del País Vasco (en adelante CAPV).

Con todo ello, el objetivo del trabajo fue actualizar, mediante censos poblacionales, el conocimiento sobre estas especies de flora incluidas en el CVEA y de interés localizadas en la ZEC del Río Zadorra dentro del término municipal de Vitoria-Gasteiz.

\begin{tabular}{|l|l|l|l|l|}
\hline \multicolumn{1}{|c|}{ Taxon } & BOPV/EHAA, 2011 & $\begin{array}{c}\text { Aizpuru et al., } \\
2010\end{array}$ & $\begin{array}{c}\text { Directiva } \\
\text { 92/43/CEE }\end{array}$ & \multicolumn{1}{|c|}{ Localidad } \\
\hline Berula erecta & Peligro Extinción & En Peligro & & Estarrona \\
\hline Himantoglossum hircinum & Rara & Casi Amenazada & $\begin{array}{l}\text { Gamarra- } \\
\text { Abechuco }\end{array}$ \\
\hline Narcissus minor subsp.exiguus & Interés Especial & Interés Especial & Anexo II & Desconocida \\
\hline $\begin{array}{l}\text { Narcissus pseudonarcissus } \\
\text { subsp.pallidiflorus }\end{array}$ & Rara & Casi Amenazada & Anexo II & Amarita \\
\hline Pentaglottis sempervirens & Vulnerable & Vulnerable & & Amarita \\
\hline Ranunculus auricomus & Vulnerable & Vulnerable & & Amarita \\
\hline
\end{tabular}

Tabla 1.- Figuras de protección de las especies estudiadas y localidades conocidas incluidas en el área de estudio previas a este trabajo..

Table 1.- Categories of protection of the species studied and known sites included in the study area prior to this survey. 


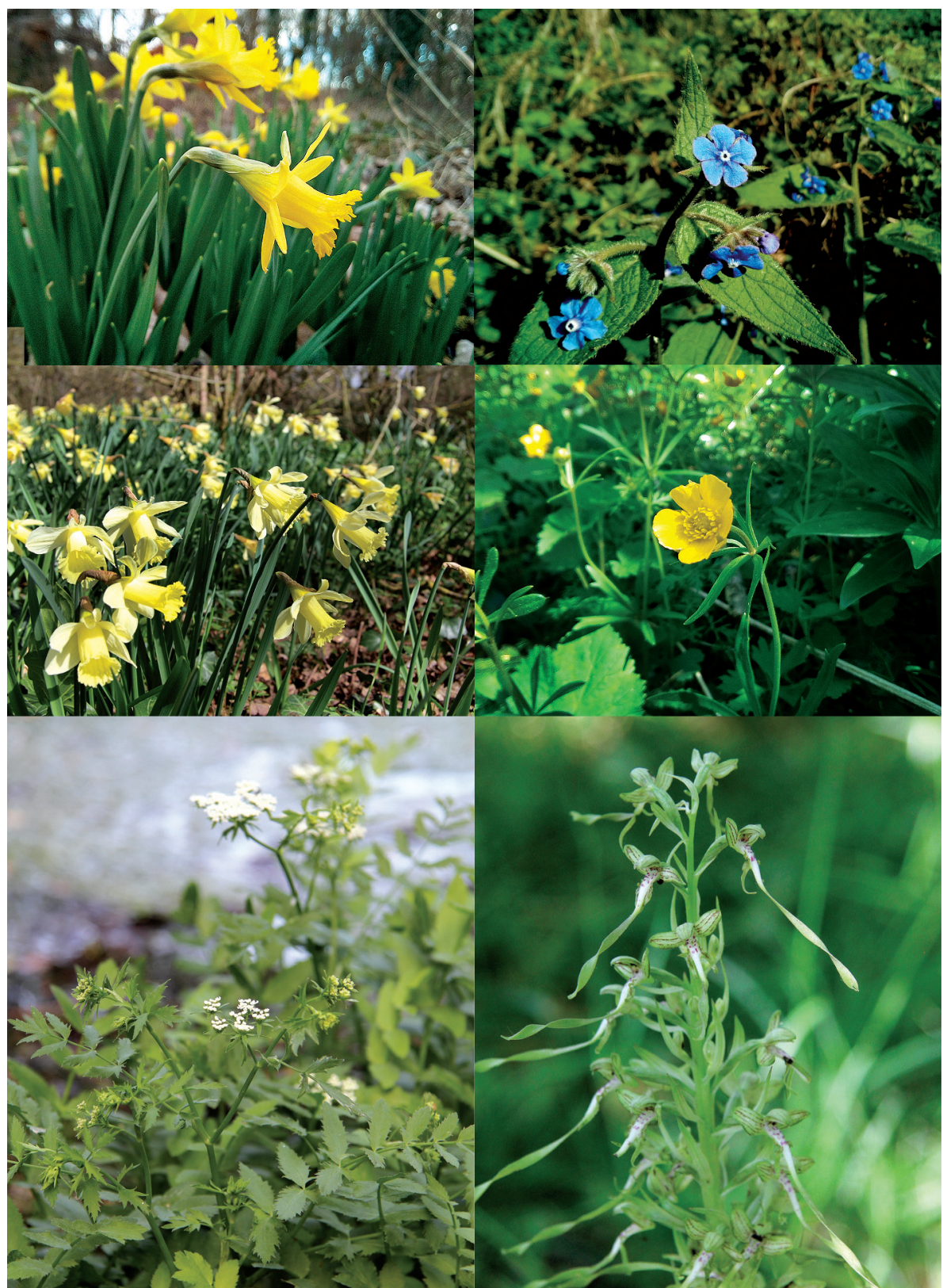

Fig. 2.- De arriba a abajo y de izquierda a derecha: Narcissus minor subsp. exiguus, Narcissus pseudonarcissus subsp. pallidiflorus, Berula erecta, Pentaglottis sempervirens, Ranunculus auricomus, Himantoglossum hircinum.

Fig. 2.- From top to bottom and from left to right: Narcissus minor subsp. exiguus, Narcissus pseudonarcissus subsp. pallidiflorus, Berula erecta, Pentaglottis sempervirens, Ranunculus auricomus, Himantoglossum hircinum. 
Las poblaciones de flora objeto de estudio en esta área eran conocidas por el Banco de Germoplasma del Jardín Botánico de Olarizu, y habían sido visitadas durante estudios anteriores de conservación ex situ de flora del municipio de Vitoria-Gasteiz (Agut, 2012). Estas poblaciones fueron descritas por los trabajos botánicos previos de Uribe-Echebarría (2010a, 2010b), y a su vez están citadas en el Documento de información ecológica y objetivos de conservación de la ZEC (Tabla 1). Así mismo se revisaron los datos referentes a estas especies en Anthos-Sistema de Información de las Plantas de España (2017) y en el Portal de datos GBIF (2017), pese a que este último portal no aportó datos nuevos.

Por otro lado, se llevaron a cabo prospecciones de campo en las áreas y hábitats adecuados para albergar la presencia de estas especies con el objetivo de comprobar la presencia o ausencia de cada una de ellas más allá de las poblaciones conocidas.

Todos los núcleos poblacionales de las especies objeto de estudio fueron georreferenciados. Las cuadrículas UTM de $1 \mathrm{~km}$ de lado indicadas en el trabajo corresponden al huso 30T, por lo que este dato se ha omitido en las citas, y el sistema de referencia corresponde al Datum ETRS89. De cada nueva localización de las especies se herborizó un pliego de herbario testigo, que fue incluido en el Herbario OLA del Jardín Botánico de Olarizu (OLA). La nomenclatura de las especies es la propuesta en Aizpuru et al. (1999), a excepción de Narcissus minor subsp. exiguus, descrito siguiendo la nomenclatura propuesta en Agut et al. (2018).

Los censos poblacionales se llevaron a cabo utilizando el procedimiento propuesto en la Metodología del Atlas de Flora Amenazada de España (AFA) (Iriondo, 2011). De tal manera que los censos poblaciones de todas las especies, a excepción de Berula erecta, se realizaron mediante estimación directa hasta que se sobrepasaron los 2.500 ejemplares. En cambio, debido a que Berula erecta es una especie estolonífera y que en ocasiones permanece bajo el curso de agua, el censo se realizó mediante el área de ocupación de la especie.

En el caso de Pentaglottis sempervirens, debido a las condiciones del terreno y la densidad de plantas en algunos núcleos, con el objetivo de evitar el excesivo pisoteo del hábitat y no dañar a los ejemplares en estos núcleos de mayor densidad, se contabilizaron los tallos floríferos de la especie durante la época de floración.

A continuación, se presentan las novedades y los resultados de los censos realizados para cada una de las especies estudiadas.

\section{Berula erecta (Hudson) Coville}

Una de sus 3 poblaciones conocidas en la CAPV se localiza al sur del municipio, en la confluencia del río Zalla con el río Zadorra. Uribe-Echebarría (2010a) recoge la cita de 
Patino et al. (1992) para esta población, y describe, tras su observación in situ, que la población ocupaba $4 \mathrm{~m}^{2}$ con pocos individuos en una zona de un vado pedregoso (WN1944).

En 2011 y 2016 se comprobó que la población se extendía aguas arriba del río Zalla, en una zona de vado pedregoso, por encima del nivel del agua durante la época de estiaje, alcanzando la cuadrícula WN1945, dato novedoso para la CAPV. En total se contabilizaron 29 núcleos poblacionales de entre 4 y 6,25 $\mathrm{m}^{2}$, además de observar algunos individuos aislados (Figura 3; Tabla 2; Agut et al., 2012; Somoza et al., 2013).

Hay que destacar que, pese a que el Documento de Medidas de Gestión de la ZEC contempla la presencia de la especie en el "ámbito" de la ZEC, ni la cuadrícula UTM conocida inicialmente, ni la nueva, se hallan estrictamente en los límites de la misma, sino muy próximos, ya que el río Zalla no se encuentra incluido en este espacio. En este aspecto, cabe señalar que la posibilidad de ampliar la ZEC e incluir dentro de sus límites el tramo bajo del río Zalla como ya se planteó previamente a la declaración de la ZEC, es una cuestión de especial relevancia en relación con la conservación de esta especie catalogada como "En Peligro de Extinción" que se debería reconsiderar.

\section{Himantoglossum hircinum (L.) Sprengel}

Orquídea rara tanto en el municipio de Vitoria-Gasteiz como en la Comunidad Autónoma del País Vasco, cuyas poblaciones son generalmente pequeñas, aisladas y con reducido número de individuos. La única localidad considerada en el Documento de información ecológica y objetivos de conservación de la ZEC (Gobierno Vasco, 2014) se refiere a la población de Lapuebla de Arganzón (Condado de Treviño, Burgos).

En 2013, gracias a las observaciones fruto del Programa de Seguimiento de Orquídeas del Municipio de Vitoria-Gasteiz promovido por el Jardín Botánico de Olarizu y el Centro de Estudios Ambientales del Ayuntamiento de Vitoria-Gasteiz, se puso de manifiesto la existencia de 1 nueva localidad en un lastonar a la sombra del arbolado, en el tramo de Gamarra-Abechuco del parque fluvial del Zadorra perteneciente al Anillo Verde de Vitoria-Gasteiz, incluido en la ZEC (Figura 3; Agut, 2013, 2014, 2015, 2016, 2017). Desde entonces hasta la actualidad, gracias a las visitas periódicas al lugar, se ha seguido observando el ejemplar, que sufre una fuerte presión recolectora de su parte aérea, la cual es cortada año tras año durante la época de floración por algunos de los habituales paseantes.

En consecuencia, desde el Banco de Germoplasma del Jardín Botánico de Olarizu, más allá de la gestión de las siegas respetando el periodo de floración y fructificación de las orquídeas, como ya se practica en otros puntos del Anillo Verde de Vitoria-Gasteiz con poblaciones de orquídeas de interés, se propuso como principal medida de con- 
servación in situ la instalación de un balizamiento que señalara la presencia de la especie con el fin de informar de su presencia y su protección legal para evitar dicha presión recolectora.

Narcissus pseudonarcissus L. subsp. pallidiflorus (Pugsley) A. Fernandez

El Documento de información ecológica y objetivos de conservación de la ZEC (Gobierno Vasco, 2014) recoge las citas de Uribe-Echebarría (2010a) para Narcissus pseudonarcissus, quien señalaba su presencia en Amarita, arroyo Santa Engracia, en 4 cuadrículas UTM 1x1 km (WN3050, WN2950, WN2951, WN3051).

Además de constatar su presencia en estas cuadrículas, la especie fue localizada en dos nuevas cuadrículas UTM 1x1 km (WN2952, WN2949) en las cuales no estaba citada por trabajos previos, y que por lo tanto suponen una novedad en el conocimiento de la distribución de esta especie en la ZEC. Ambas cuadrículas delimitan la distribución de la especie en el área de estudio (Figura 3). La primera por el sur, y cuenta únicamente con un pequeño núcleo poblacional con 70 individuos (WN2949, Pliego testigo: OLA-627) en la confluencia del rio Santa Engracia con el Zadorra. La segunda por el norte, coincidiendo con las inmediaciones del puente de la Autopista AP-1(WN2952, Pliegos testigo: OLA-625 y OLA-629), y cuenta con más de 1.500 ejemplares (Tabla 2).

A través de estas 6 cuadrículas UTM, la especie aparece de manera dispersa por el bosque de ribera del río Santa Engracia, contabilizándose únicamente 2 núcleos poblacionales con un tamaño superior a los 2500 individuos (Tabla 2): uno se corresponde con el robledal de Amarita y el otro se localiza en un pequeño meando del río, aguas arriba del robledal, el cual conserva una pequeña superficie del bosque de ribera.

Narcissus minor L. subsp. exiguus (Salisb.) Agut, Garaikoetxea, González, Jáñez, Solis \& Urrutia.

El Documento de información ecológica y objetivos de conservación de la ZEC recoge las citas de la Lista Roja de la flora vascular de la CAPV (Aizpuru et al., 2010) para este taxón en las localidades de Iruña de Oca, Villodas, Trespuentes y Mendibil, próximas a la ZEC. Sin embargo, no se recoge ninguna cita en el ámbito del área de estudio.

Fruto de este trabajo, se localizó la presencia puntual de la especie en las siguientes 3 localidades desconocidas hasta la fecha, las cuales suponen una novedad tanto para la ZEC en su totalidad como para el municipio de Vitoria-Gasteiz (Figura 3; Tabla 2):

1.- Mendibil (WN3050, límite municipal de Vitoria-Gasteiz con Arrazua-Ubarrundia). Anthos (2017) recoge la cita de Uribe-Echebarría (1998) para esta especie en 


\begin{tabular}{|c|c|c|c|}
\hline Taxon & $\begin{array}{c}\text { Presencia cuadricula } \\
\text { UTM } 1 \times 1 \mathrm{Km}\end{array}$ & $\begin{array}{c}\text { Núcleos } \\
\text { poblacionales }\end{array}$ & $\mathrm{N}^{\circ}$ ejemplares \\
\hline \multirow{2}{*}{ Berula erecta } & 30TWN1944 & 17 & $\begin{array}{l}\text { Individuos aislados y } \\
\text { masas de hasta } 6.25 \mathrm{~m}^{2}\end{array}$ \\
\hline & 30TWN1945 & 12 & $\begin{array}{l}\text { Individuos aislados y } \\
\text { masas de hasta } 4 \mathrm{~m}^{2}\end{array}$ \\
\hline Ranunculus auricomus & $30 T W N 2950$ & 2 & 204 \\
\hline Pentaglotis sempervirens & $30 T W N 2950$ & 8 & $\begin{array}{l}139 \text { tallos floríferos y } 87 \\
\text { rosetas basales sin tallos } \\
\text { floríferos }\end{array}$ \\
\hline \multirow{5}{*}{$\begin{array}{l}\text { Narcissus pseudo narcissus } \\
\text { subsp. pallidiflorus }\end{array}$} & $\begin{array}{l}30 \text { TWN } 3050 \\
30 \text { TWN } 2950\end{array}$ & 1 & $>2500$ \\
\hline & 30 TWN 2951 & 20 & $>2500$ \\
\hline & 30 TWN 3051 & 1 & 21 \\
\hline & 30 TWN 2952 & 18 & 1759 \\
\hline & 30 TWN 2949 & 1 & 70 \\
\hline \multirow{3}{*}{$\begin{array}{l}\text { Narcissus minor } \\
\text { subsp. exiguus }\end{array}$} & 30TWN3050 & 1 & 78 \\
\hline & $30 T W N 2446$ & 1 & 1 \\
\hline & 30TWN2951 & 1 & 12 \\
\hline \multirow{2}{*}{ Narcissus $x$ petri-mariae } & $30 T W N 2951$ & 1 & 2 \\
\hline & 30TWN2950 & 1 & 1 \\
\hline Himantoglossum hircinum & 30T WN2746 & 1 & 1 \\
\hline
\end{tabular}

Tabla 2.- Localización y resultados de los censos poblacionales de las especies objeto de estudio. Table 2.- Location and results of population censuses of species included in the survey.

esta cuadrícula, pero fuera de los límites de la ZEC, por lo que hasta la fecha no se tenía constancia de su presencia en el área de estudio. La especie se localiza en 2 pequeños núcleos, uno en cada orilla del río Zadorra. El primero apenas ocupa una superficie de $3 \mathrm{~m}^{2}$ y consta de 60 individuos reproductores. El segundo, solo contiene 18 individuos en una superficie de $2 \mathrm{~m}^{2}$. Ambos puntos presentan problemas de contaminación por vertidos y basuras (plásticos y otros tipos de residuos procedentes de la actividad humana), debido a su proximidad a caminos bastante transitados. En ambos casos los ejemplares son de gran tamaño, mayor de lo habitual (Pliego testigo: OLA-679).

2.- Parque fluvial del río Zadorra (WN2446, Anillo Verde de Vitoria-Gasteiz).Se observó la presencia de un único ejemplar florecido en este punto. 


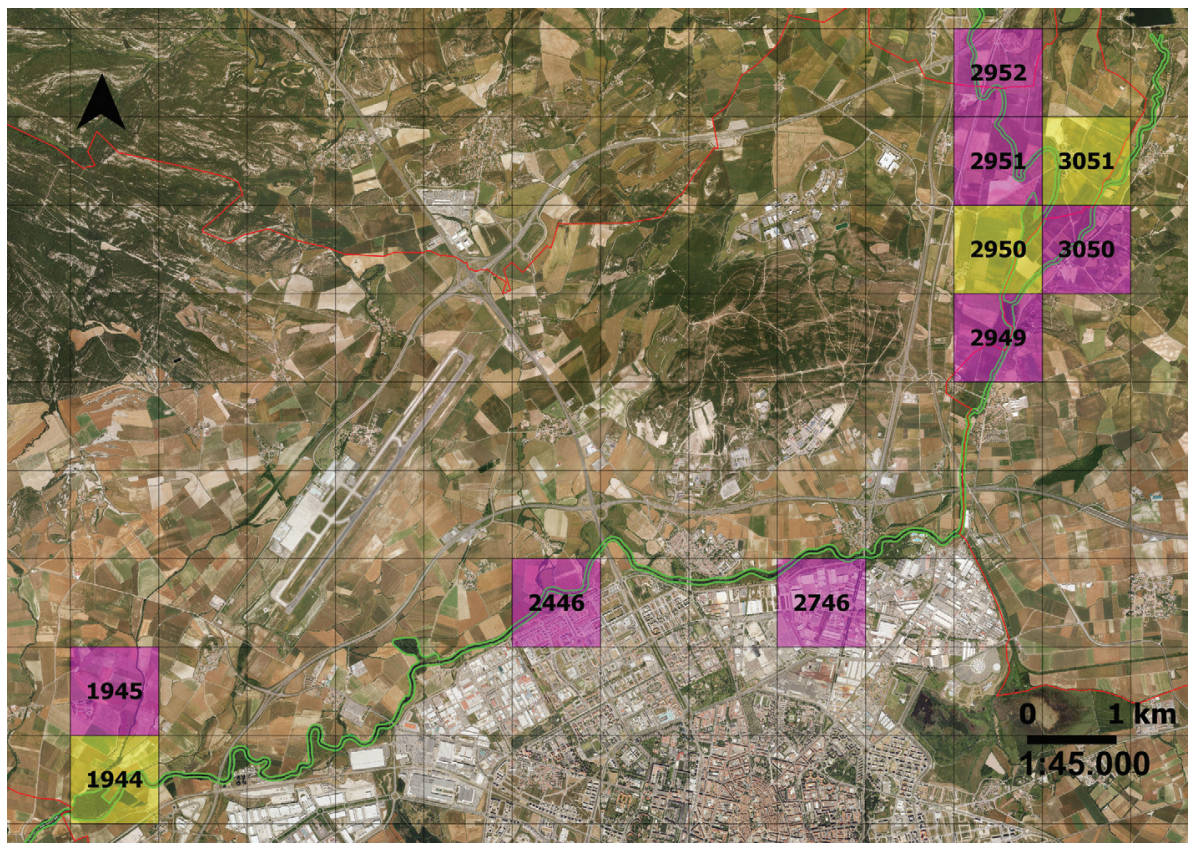

Fig. 3.- Cuadrículas UTM 1x1 km con presencia de las especies objeto de estudio. En amarillo: localidades conocidas previas al presente trabajo. En morado: nuevas localidades.

Fig. 3.- $1 \times 1 \mathrm{~km}$ UTM grid squares with the presence of the species dealt with in the survey. In yellow: known sites prior to this survey; in purple, new sites.

3.- Río Santa Engracia (WN2951). Se contabilizaron 12 individuos dispersos, entre los individuos de Narcissus pallidiflorus y Narcissusx petri-mariae, en un muy pequeño herbazal al borde del bosque de ribera.

\section{Narcissus $\boldsymbol{x}$ petri-mariae Fern. Casas}

Pese a no encontrarse incluido en el CVEA, debido al bajo número de localidades conocidas de este híbrido en la CAPV, es conveniente destacar su presencia en el área de estudio.

Además de la reciente cita de Pello Urrutia en el Herbario Digital Xavier de Arizaga (VV.AA., 2017) para este híbrido en el robledal de Amarita (pliego HDXA 6084), se localizó un nuevo núcleo poblacional con 2 ejemplares (Pliego testigo: OLA 680) compartiendo hábitat con N. pallidiflorus y N. minor subsp. exiguus, en un punto cercano al robledal, en la ribera del río Santa Engracia (Figura 3). Este hallazgo supone una novedad tanto para el híbrido, del que se conocen muy pocas localidades, como para la ZEC y el municipio de Vitoria-Gasteiz. 
Una mejora del estado de conservación del bosque de ribera y sus herbazales en este río resultaría fundamental para el desarrollo y conservación de estos narcisos, y en especial para Narcissus minor y Narcissus $x$ petri-mariae, los cuales cuentan con un número bajo de ejemplares en el área de estudio, el primero debido a la ausencia de hábitats óptimos para su desarrollo, se trata de una planta fuera de estación en este caso, mientras que el segundo podría tratarse de un hábitat adecuado pero tampoco óptimo.

\section{Pentaglottis sempervirens (L.) L.H. Bailey}

Especie que únicamente dispone de 2 poblaciones en la CAPV, una de ellas en la parte occidental de Bizkaia, en Sopuerta (Aizpuru et al., 2010) y la otra en el área de estudio, en el robledal de Amarita.

Esta población supone el límite oriental conocido de la especie en la Península Ibérica. Se conoce desde los años 80 del siglo pasado, cuando fue citada por Uribe-Echebarría y Alejandre (1982). Desde esta fecha hasta la actualidad, la población se mantiene estable, pese a que el número de plantas florecidas varía de unos años a otros. Según datos de la Lista Roja de la Flora del País Vasco (Aizpuru et al., 2010) en el trabajo de Campos \& Silván (2001) se contabilizaron124 ejemplares, de los que 43 eran reproductores mientras que en el estudio de Garmendia (2006) se contabilizaron 125 ejemplares, 55 de ellos en flor. A su vez, en el año 2009, Uribe-Echebarria (2010a) señaló un total de 170 plantas, 90 de ellas en flor y 80 vegetativas.

Cada planta produce entre 3 y 5 tallos floríferos (Uribe-Echebarría, 2010a). Durante el mes de mayo de 2017 se contabilizaron 139 tallos floríferos y 87 rosetas basales sin tallos floríferos dentro del robledal (Tabla 2). Es destacable el hecho de que 110 de estos tallos se encuentran en el margen del robledal, colindando con una finca de cultivo de cereal, en un área que recorre una franja de 25 x 5 m y recibe una mayor insolación. Los individuos de este núcleo poblacional son los de mayor tamaño de la población. Sin embargo, las prácticas agrarias de la finca colindante, como el uso de fitosanitarios/herbicidas y las labores de labranza, que prácticamente alcanzan a este núcleo, suponen importantes factores de amenaza. Mientras tanto, dentro del robledal, la planta presenta individuos de menor tamaño y muy dispersos, formando pequeños núcleos con un número de individuos siempre inferior a la docena.

\section{Ranunculus auricomus $\mathrm{L}$.}

Especie rara en la CAPV, donde se localiza en los robledales éutrofos de fondo de valle de Quercus robur (Crataego laevigatae-Quercetum roboris), en las comarcas de valles subatlánticos de Urkabuztaiz, Kuartango y la Llanada Alavesa y cuyas poblaciones poseen un número muy bajo de individuos (Uribe-Echebarría et al., 2006).La única 
población existente en la ZEC es la citada por Uribe-Echebarría (2010a), el cual señaló la presencia de 12 individuos florecidos en el interior del robledal de Amarita, en una zona de sendero poco transitado.

En abril de 2017, se llevó a cabo el censo poblacional en este lugar, contabilizándose 200 individuos en flor, formando un núcleo de unos $30 \mathrm{~m}^{2}$ (Tabla 2). A su vez, en julio de 2017 se contabilizaron 4 plantas en fructificación en otro punto del mismo robledal, en el borde de un camino bastante transitado.

De modo que, desde 2010 hasta la actualidad se ha observado un aumento de ejemplares de esta población. Este aumento podría tener cierta relación con las visitas al bosque realizadas durante diferentes trabajos botánicos y naturalísticos en los últimos años. Este hecho podría haber provocado que el pisoteo no excesivo impidiera el desarrollo en exceso del zarzal y/o la orla arbustiva, factores que podrían perjudicar al desarrollo de R. auricomus, tal y como señalaba Uribe-Echebarría (2010a).

Tanto Pentaglottis sempervirens como Ranunculus auricomus, son especies con un número muy bajo de poblaciones en la CAPV y de reducido número de ejemplares, para las que resulta fundamental evaluar periódicamente sus tendencias poblacionales con el fin de establecer las medidas y actuaciones necesarias para su conservación ex situ e in situ, resultando de gran interés las acciones que se están llevando a cabo desde el Banco de Germoplasma del Jardín Botánico de Olarizu. Entre estas acciones podemos destacar la recolección y conservación de germoplasma de estas especies, así como su reproducción y cultivo ex situ, el cual ha permitido llevar a cabo las primeras actuaciones de conservación in situ como los reforzamientos de proximidad (Laguna \& Ferrer, 2012) realizados para estos taxones en la ZEC.

\section{Agradecimientos}

A Beatriz Tellechea Puente, por su ayuda inestimable en la realización de algunos de los censos poblacionales llevados a cabo en el presente trabajo.

A Pello Urrutia, del Instituto Alavés de la Naturaleza, por mostrarnos in situ en el bosque de Amarita el ejemplar de Narcissus x petri-mariae que había localizado y nos permitió identificar correctamente el que descubrimos más tarde.

A Antonio González, del Instituto Alavés de la Naturaleza, por acompañarnos durante varias visitas al bosque de Amarita a lo largo del año.

La mayor parte de los resultados de este estudio fueron fruto del proyecto "Conservación y refuerzo poblacional de la flora amenazada del Río Zadorra (ES2110010) en el municipio de Vitoria-Gasteiz" subvencionado por el Dpto. de Medio Ambiente, Planificación Territorial, Agricultura y Pesca del Gobierno Vasco. 


\section{Bibliografía}

Agut, A. 2012. El Banco de Germoplasma Vegetal del Jardín Botánico de Olarizu. Biogaia, 6-7.

Agut, A., Del Canto, A., Mira, S. 2012. Memoria final del proyecto programa de conservación y reintroducción de la flora amenazada y característica de los robledales isla de la Llanada Alavesa. Informe inédito realizado por el Banco de Germoplasma Vegetal del Jardín Botánico de Olarizu para el Gobierno Vasco. Vitoria-Gasteiz.

Agut, A. 2013. Programa de seguimiento de las poblaciones de orquídeas del anillo verde de Vitoria-Gasteiz. Dpto. de Medio Ambiente y Espacio Público. Ayuntamiento de VitoriaGasteiz.

Agut, A. 2014.Programa de seguimiento de las poblaciones de orquídeas del anillo verde de Vitoria-Gasteiz. Dpto. de Medio Ambiente y Espacio Público. Ayuntamiento de VitoriaGasteiz.

Agut, A. 2015.Programa de seguimiento de las poblaciones de orquídeas del anillo verde de Vitoria-Gasteiz. Dpto. de Medio Ambiente y Espacio Público. Ayuntamiento de VitoriaGasteiz.

Agut, A. 2016.Programa de seguimiento de las poblaciones de orquídeas del anillo verde de Vitoria-Gasteiz. Dpto. de Medio Ambiente y Espacio Público. Ayuntamiento de VitoriaGasteiz.

Agut, A. 2017.Programa de seguimiento de las poblaciones de orquídeas del anillo verde de Vitoria-Gasteiz. Dpto. de Medio Ambiente y Espacio Público. Ayuntamiento de VitoriaGasteiz.

Agut, A., Garaikoetxea, F., González, A., Jáñez, A., Solís, J.P. y Urrutia, P. 2018. Sobre Narcissus asturiensis (Jord.) Pugsley subsp. brevicoronatus (Pugsley) Uribe-Echebarría (Amaryllidaceae) y su ubicación en el grupo asturiensis-minor. Flora Montiberica 70: 50-66.

Aizpuru, I., Aseginolaza, C., Uribe-Echebarría, P.M., Urrutia, P., Zorrakin, I. 1999. Claves ilustradas de la flora del País Vasco y territorios limitrofes. Servicio Central de Publicaciones del Gobierno Vasco. Vitoria-Gasteiz.

Aizpuru, I., K. Alvarez, J. Balentzia, I. Biurrun, J.A. Campos, I. García, J. Garmendia, M. Herrera, L. Oreja, S. Patino, A. Prieto, I. Tamayo, P.M. Uribe-Echebarría. 2010. Lista roja de la flora vascular de la CAPV. Dpto. De Medio Ambiente, Planificación Territorial, Agricultura y Pesca. Gobierno Vasco.

Anthos.2011 [2017]. Information System of the plants of Spain. Real Jardín Botánico, Consejo Superior de Investigaciones Científicas, Fundación Biodiversidad. Recurso electrónico en www.anthos.es. Consulta [18 de enero 2017].

BOPV/EHAA. 2011. Orden 10 de Enero de 2011, de la Consejera de Medio Ambiente, Planificación Territorial, Agricultura y Pesca, por la que se modifica el Catálogo Vasco de Especies Amenazadas de la Fauna y Flora Silvestre y Marina y se aprueba el texto único. Departamento de Medio Ambiente, Planificación Territorial, Agricultura y Pesca del GobiernoVasco. Vitoria-Gasteiz. 
Campos, J.A, Silván, F. 2001. Estudio de la situación de Pentaglottis sempervirens (L.) L.H. Bailey en la Comunidad Autónoma del País Vasco. Eusko Jaurlaritza.

Directiva 92/43/CEE del Consejo, de 21 de mayo de 1992, relativa a la Conservación de los Hábitats Naturales y de la Fauna y Flora silvestres.

Garmendia, J. 2006. Estudio de la flora en peligro de extinción en la Comunidad Autónoma del PaísVasco: distribución, estado y propuestas de conservación. Informe inédito realizado por la Sociedad de Ciencias Aranzadi para el Gobierno Vasco.

GobiernoVasco/Eusko Jaurlaritza. 2014.Documento de objetivos y medidas de conservación para la declaración de la Zona Especial de Conservación ES2110010 Río Zadorral Zadorra Ibaia. Dirección del Medio Natural y Planificación Ambiental del Departamento de Medio Ambiente y Política Territorial del Gobierno Vasco.

GBIF. org (2017), Página de Inicio de GBIF. Disponible en: https://www.gbif.net [18 de enero de 2017].

Iriondo, J.M., Coord. 2011. Atlas y Libro Rojo de la Flora Vascular Amenazada de España. Manual de metodología del trabajo corológico y demográfico. Dirección General de Medio Natural y Política Forestal (Ministerio de Medio Ambiente, y Medio Rural y Marino). Sociedad Española de Biología de la Conservación de Plantas. Madrid.

Laguna, E., Ferrer, P.P. 2012. Reforzamiento de proximidad y neopoblaciones de seguridad, nuevos conceptos complementarios para determinados tipos de implantaciones vegetales in situ. Conservación Vegetal 16-14.

Patino, S., Uribe-Echebarría, P.M., Urrutia, P., Valencia, J. 1992. Notas corológicas sobre la flora vascular del País Vasco y aledaños. Est. Mus. Cienc. Nat. Alava 6: 57-67.

Somoza, E., Agut, A., Salas, N., Loidi, J. 2013. Bases científicas para la conservación de Betula erecta (Huds.) Coville, una planta en peligro de extinción en la Comunidad Autónoma del País Vasco. VI Congreso de Biología de Conservación de Plantas. Murcia. 15-18 de Octubre de 2013.

Uribe-Echebarría, P.M., Alejandre, J.A. 1982. Aproximación al catálogo florístico de Álava. J.A. Alejandre. Vitoria-Gasteiz.

Uribe-Echebarría, P.M. 1998. Sobre el grupo Narcissus asturiensis (Jordan) Pugsley (AmaryIlidaceae) en la Península Iberica. Est. Mus. Cienc. Nat. de Alava 13: 157-166.

Uribe-Echebarría, P.M. 2010a. La flora amenazada del municipio de Vitoria-Gasteiz (Álava). Centro de Estudios Ambientales. Vitoria-Gasteiz.

Uribe-Echebarría, P.M. 2010b. Estudio de la caracterización botánica de los bosques de fondo de valle del municipio de Vitoria. Gasteiz (Álava). Centro de Estudios Ambientales. VitoriaGasteiz.

Uribe-Echebarría, P.M., Zorrakin, I., Campos. J.A., Dominguez, A. 2006. Flora vascular amenazada de la Comunidad Autónoma del País Vasco. Servicio Central de Publicaciones del 
Gobierno Vasco. Departamento de Medio Ambiente y Ordenación del Territorio del Gobierno Vasco. Vitoria-Gasteiz.

V.AA. 2017. Herbario Digital Xavier de Arizaga. Disponible en: http://www.herbario.ian-ani.org. 